\title{
An Ontology-based Method of Knowledge Modelling for Remanufacturing Process Planning
}

3 Yan $\mathrm{He}^{\mathrm{a}}$, Chuanpeng Hao ${ }^{\mathrm{a}}$, Yulin Wang*b ${ }^{* \mathrm{~b}}$, Yufeng $\mathrm{Li}^{* \mathrm{a}}$, Yan Wang ${ }^{\mathrm{c}}$, Lingyu Huang ${ }^{\mathrm{d}}$,

Xiaocheng $\operatorname{Tian}^{\mathrm{a}}$

5 a State Key Laboratory of Mechanical Transmission, Chongqing University, Chongqing, 6 400030, China

7 b School of Mechanical Engineering, Nanjing University of Science and Technology, Nanjing,

8 210094, China

$9{ }^{\mathrm{c}}$ Department of computing, Engineering and Mathematics, University of Brighton, Brighton,

10 BN2 4GJ, United Kingdom

$11{ }^{\mathrm{d}}$ Department of Mechanical and Energy Engineering, South University of Science and

12 Technology, Shenzhen,518000, China

$13 *$ Corresponding author. State Key Laboratory of Mechanical Transmission, Chongqing 14 University, Chongqing 400030, China (Yufeng Li). School of Mechanical Engineering, Nanjing 15 University of Science and Technology, Nanjing, 210094, China (Yulin Wang)

16 E-mail address: liyufengcqu@cqu.edu.cn (Yufeng Li, Tel: +86 17783903035),

17 wyl_sjtu@126.com (Yulin Wang, Tel: +86 13451851103)

18 Abstract:

19 Remanufacturing that returns used products to a like-new condition with equivalent 20 warranty to match is an emerging triple-win (environmental, economic and social) 21 industry. Process planning plays a vital role in the success of remanufacturing. However, 22 compared with traditional mass manufacturing, the design of remanufacturing process 23 planning (RPP) is far more complex and time-consuming, heavily depending on the 24 experiences of operators. Since each returned used product, namely the raw materials 25 for remanufacturing, is different, a customized RPP tackling the individuality of 26 returned used products is essential. To this end, the reuse of remanufacturing knowledge 27 from past successful RPP could lead to efficient generation of new process planning for 28 new arrivals. This paper proposes an ontology-based method for knowledge modelling 29 for RPP rapidly. In this method, (1) remanufacturing-ontology provides a unified 30 framework for the management of information and knowledge from various sources. 31 Especially, the remanufacturing knowledge modelling including problem description 
1 and problem solution is constructed via a remanufacturing semantic model; (2) Case-

2 Based Reasoning (CBR) method is applied to reuse the knowledge from the most

3 similar previous successful remanufacturing case for the rapid generation of RPP,

4 leading to considerable time and cost saving. An application program is also presented

5 to realize the proposed method. In addition, a case study of crankshaft remanufacturing

6 is carried out to verify the feasibility and efficiency of the proposed method.

7 Keywords: Ontology, Knowledge modelling, Knowledge reuse, Remanufacturing process planning, 8 CBR

\section{1. Introduction}

Remanufacturing is defined as "a process of returning a used product to at least its

11 original performance with a warranty that is equivalent to or better than that of the 12 newly manufactured product” by British Standard BS8887 (2009). As a crucial End13 Of-Life (EOL) activity within the circular economy, it can release the residual value of 14 used products (e.g., automotive parts and machine tools) compared to simply materials 15 recycling. The remanufacturing industry is regarded as an eco-industry, as it not only 16 alleviates environmental contamination but also reduces energy consumption and $\mathrm{CO}_{2}$ 17 emission ( Liu et al., 2014; Feng et al., 2016; Jiang et al., 2019). For example, the 18 remanufacturing of automotive components yields around $88 \%$ materials savings 19 compared to that of a new product, with an associated $53 \%$ decrease in $\mathrm{CO}_{2}$ emission 20 and $56 \%$ less energy consumption (Parker et al., 2015). In addition, from the economic 21 and social perspectives, remanufacturing could satisfy consumers' needs (usually as22 new quality with a lower price), create new job opportunities and new markets, 23 improved customer relation and satisfaction, and build a favorable image for companies 24 (Ismail et al., 2017).

25 Despite the tremendous benefits offered by remanufacturing, the 26 remanufacturability largely depends on process planning, which bridges returned used 27 products and remanufactured products. It contains the design of process route, sequence 28 and parameters, and the associated resources planning, e.g., operators, facilitate, tools 29 and time etc. for remanufacturing. A well-designed RPP could lead to enhanced 30 remanufacturing rate, improved product quality, and reduced capital investment costs 31 (Jiang et al., 2014; Zhou et al., 2014). However, compared to traditional mass 32 production, the design of RPP suffers from a high level of uncertainty imposed by 
1 varying quality and quantity of the returned products, often called cores, that were

2 subjected to different operation environments, service life, maintenance measures, and

3 reverse logistics (Wang et al., 2019; Peng et al., 2019). Thus, a tailored RPP is required

4 for each core which is a time-consuming, labor-intensive and error-prone process (Jiang

5 et al., 2019). The design of RPP heavily depends on the skills and experience of RPP

6 designers since it is often a trial-and-error manual operation, leading to costly and

7 lengthy RPP generation, a major barrier for the uptake of remanufacturing. This paper

8 aims to develop a novel method to model and reuse remanufacturing domain knowledge

9 based on ontology for rapid RPP.

10 Knowledge, composed of useful information, refers to the relevant and objective 11 information gained through experience (Cooper and Paul, 2014). Essentially, RPP 12 generation is very knowledge-intensive, including knowledge acquisition of analysis of 13 design specification, failure features and understanding of capability and facility of 14 remanufacturing factory, knowledge mapping between failure cores and restoration 15 processes, decision-making in the process sequence and parameters, and 16 remanufacturing resource allocation. Hence, a knowledge modelling method is 17 paramount to make full use of remanufacturing domain knowledge including existing 18 skills and experience to generate a sound RPP and thereby increasing the success rate 19 and efficiency of remanufacturing. In addition, the different RPP may be generated by 20 different operators depending on their experiences, each with different performance. 21 Thus, it is important to record the best practices for reusing and experience sharing so 22 as to generate the optimal RPP that is cost-effective and efficient.

23 Currently, there are limited studies in the RPP from the perspective of knowledge 24 modelling. Remanufacturing knowledge may be constructed based on understanding 25 and inter-connecting of large amounts of information and data in the remanufacturing 26 domain, which guides decision-makers to make sound decisions. Unfortunately, due to 27 data silo led from different ownership in product value chain, remanufacturing relevant 28 information and data are often fragmented and disorganized generated at various 29 product life cycle stages including begin-of-life (BOL), middle-of-life (MOL) and EOL, 30 and different stakeholders including original equipment manufacturers (OEMs), 31 distributors, recyclers, quality inspection departments, remanufacturers, etc. 32 Multifarious information and data are generated along with the product lifecycle which 33 has a significant influence on the RPP generation. For instance, the materials and 
1 specification of products at BOL affect the selection of factory resources, e.g., lathe for

2 cylinder parts, milling machine for prismatic parts and surface hardness and finish will

3 have an impact on the machining parameters for surface finish and whether or not a

4 coating operation is necessary. In order to develop an efficient method to unify, manage,

5 retrieve, and reuse remanufacturing knowledge, enabling the rapid generation of RPP.,

6 two top challenges need to be addressed to establish such a knowledge-based method

7 for RPP. Firstly, due to the complexity of remanufacturing-relevant information and

8 data, the development of a generic model to unify and manage them from different

9 lifecycle stages, thus to construct an organized and interconnected knowledge system

10 for remanufacturing is paramount. Secondly, to effectively reuse the remanufacturing-

11 relevant knowledge, there is a need to develop a reasoning mechanism to retrieve and

12 reuse the stored knowledge, facilitating the rapid generation of RPP for the new coming 13 arrivals.

14 Ontology is a formal, explicit specification of a shared conceptualization, allowing 15 non-ambiguous semantic explanation to domain knowledge, therefore enabling a better 16 representation of the knowledge (Khadir, 2012). Recently, ontology-based knowledge 17 modelling capable of combining the domain knowledge with the information 18 representation has been gradually used in various domains. It offers many advantages 19 over other concept modelling technologies because it describes a formalized and shared 20 understanding of a domain in terms of classes, possible relations between things, and 21 axioms that constrain the meaning of classes and relations (Batres et al., 2007). With 22 the purpose of efficiently reusing domain knowledge for the rapid generation of RPP to 23 improve the success rate and efficiency of remanufacturing, this paper proposed an 24 ontology-based method for knowledge modelling for RPP. With the constructed 25 knowledge model, CBR method is chosen to effectively retrieve and reuse the 26 remanufacturing knowledge due to it is an intelligent method which simulates the 27 approach how human solve problems: using the solutions of similar past problems (i.e., 28 cases) to solve the new one. It can help RPP designers make decision-making by reusing 29 the existing remanufacturing knowledge for the rapid generation of RPP. The CBR 30 method is mainly composed of four steps (1) retrieving similar cases, (2) reusing the 31 retrieved cases, (3) revised the generated solution, and (4) retaining the successful cases 32 in the case base, which means the problem-solving ability of the CBR system improves 33 continuously as the number of cases increases (Chen et al., 2016). 
Through the proposed method, the following contributions that advance the

2 knowledge can be obtained:

(1) The application of ontology makes it possible to establish a generic knowledge model of RPP. Different remanufacturing-relevant information from various sources can be unified and converted to remanufacturing-relevant knowledge, thus enables the knowledge integration, reusing, and sharing, which is fundamental to tackle the 7 problems of low efficient and high cost remanufacturing.

(2) Combined with ontology, the proposed CBR method has the ability to retrieve

9 the previous successful remanufacturing cases from the case base, supporting the rapid 10 generation of RPP. Significant time and labor cost can be saved via reusing and revising 11 the retrieved case. The new generated RPP will be stored into the case base as a new 12 case, enabling the continuous improvement of the problem-solving ability of the CBR 13 system.

14 The rest of this paper is organized as follows: Section 2 provides a literature review 15 about RPP. The overall method for the RPP is presented in section 3. The information 16 processing system based on ontology and CBR system constructed in section 4 and 17 section 5 respectively. Section 6 demonstrated the developed method for the RPP using 18 the remanufacturing of crankshafts as a case study. An interface to facilitate the process 19 planning is also developed in this section; The last section presents conclusions and 20 future work.

\section{2. Literature review}

Due to the significance of RPP to remanufacturing, several contributive studies have been carried out in this field. Jiang et al. (2014) presented a decision-making method for RPP selection considering remanufacturing time, quality and cost, which combines quality function deployment (QFD) and fuzzy linear regression. Kerbaum et al. (2009) presented an approach for the design and evaluation of the remanufacturing processes in a given facility. A mixed integer programming approach is used for the optimization of the RPP from cleaning to reassembly. There is no doubt that these studies contribute greatly to the RPP. However, due to the individuality of each product

31 addressed in existing research. The generation of RPP is still trial-and-error and laborintensive. 
The existing remanufacturing experience and knowledge, especially previous

2 successful RPP cases play a key role in the efficiency of RPP, however, it is rarely

3 considered and reused in the generation of sound process planning. To reuse the

4 previous successful remanufacturing experience, and thereby to facilitate decision-

5 making, CBR has been introduced in some remanufacturing fields, which can help

6 process planners retrieve, reuse, revise the solution of past problems to solve similar

7 new problems (Kuo, 2010; Goodall, et al. 2015) proposed a method utilizing a

8 combination of case-based reasoning and probability theory to identify similarities

9 between historical data records and the product under assessment, to predict the cost

10 and risks of remanufacturing. Veerakamolmal and Gupta (2002) suggested a CBR

11 approach to automate the disassembly process planning for multiple products. Jiang, et

12 al. (2019) proposed a hybrid approach of rough set and case-based reasoning for RPP.

13 Zhou, et al. (2014) presented a CBR based method to develop a reasonable RPP for part

14 remanufacturing. In these methods, influence factors for RPP including essential

15 characteristics, failure characteristics, and remanufacturing processing characteristics

16 are identified first, and then similarities of cases are calculated to retrieve the reusable 17 similar cases for RPP.

18 In these CBR researches for RPP, knowledge is represented in the form of case, 19 which is a kind of semantic conception including problem description and problem 20 solutions. However, the performance of these traditional CBR systems are not 21 satisfactory, as the knowledge for the existing CBR is often described in a simple 22 spreadsheet, the intricate link between different types and levels of knowledge from 23 different sources and life cycle of products have been ignored, failing in dealing with 24 complexity of knowledge modelling needed for RPP (Kolodner 1993; Guo et al. 25 2013).

26 In this paper, a new approach to knowledge modelling based on ontology is 27 proposed. There are some studies of CBR based on ontology in literature. Assali et al. 28 (2009) presented an ontology-based CBR platform to diagnose the failure of gas sensors 29 installed in industrial plants. The platform can capitalize and reuse past failure 30 experiences based on ontological models describing the domain and case structures.

31 Khadir (2012) developed a CBR application for fault diagnosis of steam turbines that 32 integrated a domain knowledge modeling in an ontological form. Xie et al. (2013) 33 proposed a CBR system for hydro-generator design, and the case base in the system 
1 was constructed based on a domain ontology to improve retrieval efficiency. Chen et al.

2 (2016) proposed an ontology and CBR based automated decision-making method for

3 the disassembly of mechanical products, the method applied ontology to uniform

4 different kinds of disassembly-related knowledge from different sources and CBR are

5 used to obtain high flexible decision-making to various conditions. These researches

6 demonstrated the effectiveness of the integration of CBR with ontology, enabling

7 efficient knowledge reuse with the CBR method. Yet these studies were focused on the

8 earlier stage of the product lifecycle, e.g. design and operation and maintenance, and

9 knowledge is only based on a single life cycle stage of the product. For remanufacturing,

10 RRP takes place at a very later stage of the product lifecycle, the knowledge modelling

11 is far more complicated, and there is a need to take into consideration information from

12 different lifecycle stages that is from different sources and in various formats. This 13 requires the ontology for RPP to be highly flexible and the intricate relationship 14 between the information to be modelled accurately. This hasn't been taken into 15 consideration to the best knowledge of the authors in prior research.

\section{3. The overall research framework}

17 A novel method for RPP integrating ontology with CBR is proposed. Fig. 1 18 demonstrates the overall structure of the method. This method contains two subsystems 19 including an information processing system and CBR system. The information process 20 system is used to organize the remanufacturing knowledgebase through ontology, and 21 the CBR system is employed to utilize the knowledge base to generate RPP for new 22 incoming arrivals. The interaction of these two subsystems enables the efficient 23 generation of RPP. 


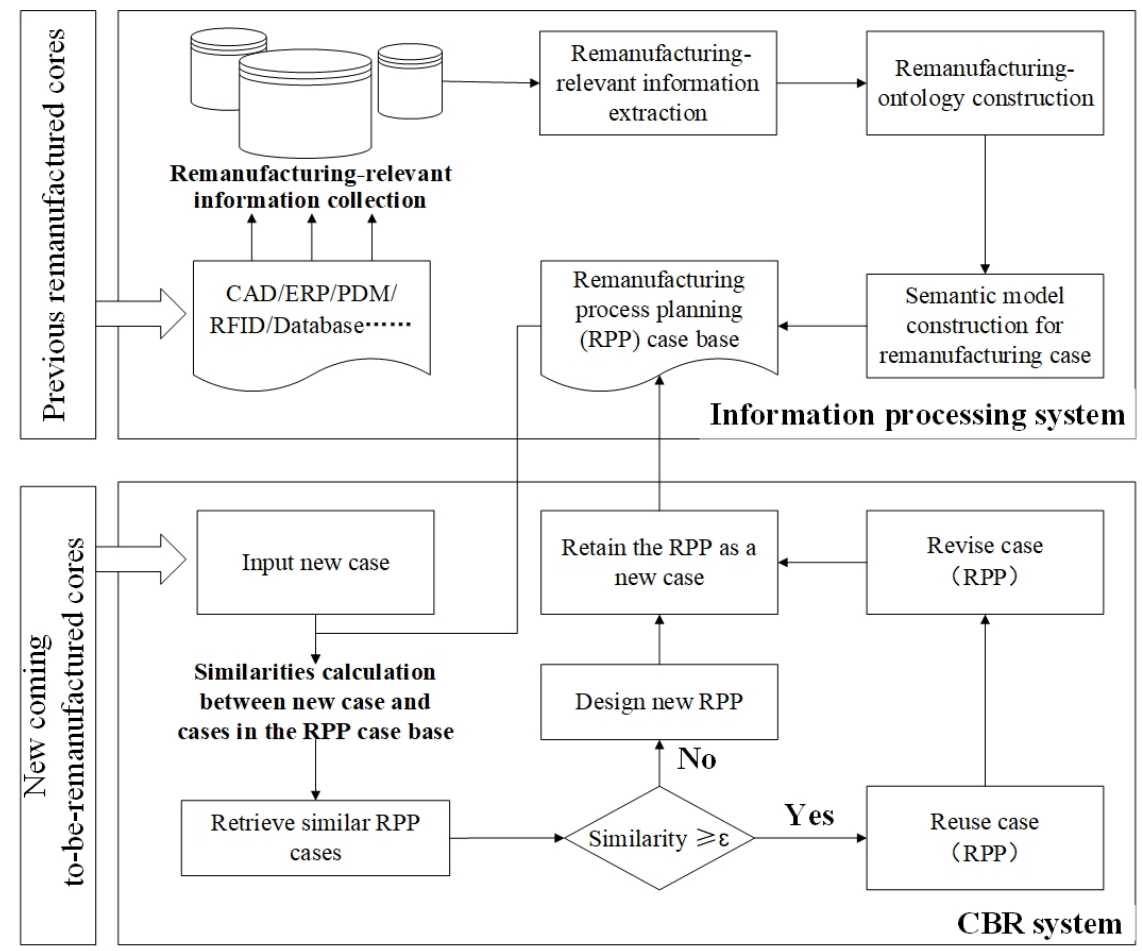

Fig. 1. Overall structure of the integrated remanufacturing process planning generation system

The purpose of the information processing system is to provide an underpinning data structure to unify heterogenous information from different sources such as CAD, Enterprise Resource Planning (ERP), Product Data Management (PDM), Radio Frequency Identification Devices (RFID), etc. The collected remanufacturing-relevant information can be finally converted to RPP cases through knowledge extraction, ontology presentation and semantic model in the system. These RPP cases describe the goal, namely what are the requirements for the remanufactured products and how to achieve the goal, in other word, how to remanufacture the EOL products back to likenew condition, which lays a foundation for the subsequent retrieval in the CBR system. The following contents explain the function of each module in this subsystem.

- Remanufacturing-relevant information collection. Based on the existing information systems, information from lifecycle stages are collected into the database.

- Remanufacturing-relevant information extraction. Redundant information can only add to the burden of the system without real value. This step identifies contributive classes of information to the presentation of RPP. Relevant useful remanufacturing information can be extracted according to the classification rules, which is essential to compose remanufacturing-relevant knowledge. 
formalized definition to the knowledge of remanufacturing domain, knowledge classes and their attribute classes are ordered by specific logical rules. All the knowledge in the processing system is expressed according to the remanufacturing-ontology.

- Semantic model construction. Based on the remanufacturing-ontology, this step maps the remanufacturing-relevant knowledge to a semantic model. It provides a generic model to describe remanufacturing cases so that an RPP case base can be constructed to store previous successful cases. It will be the basis for the subsequent CBR system.

With the constructed remanufacturing case base, the purpose of the CBR system is to reuse the knowledge stored in the case base, enabling the rapid generation of new process planning for the new cores. Normally, a CBR system is a four-step cycle process including retrieve, reuse, revise and retain after the new case has been input in the system.

- Retrieve similar remanufacturing cases. It calculates the similarities between the new case and the cases stored in the RPP case base. The higher the similarity between cases, the more similar the solutions of the cases will be, which means the solution to a similar case is beneficial to the new problem.

- Reuse case. When the similarity between the new case and the previous case is greater than a similar threshold $\varepsilon$ (e.g. $70 \%$ ), the solution to the previous case is promising to solve the new case, which means the previous RPP can be reused for the new coming cores.

- Revise case. The retrieved process planning may be revised before reusing according to the real condition of the to-be remanufactured core, if necessary.

- Retain new generated case. If a new RPP has been generated successfully, it will be retained in the RPP case base, which, in turn, can improve the problemsolving ability of the CBR system continuously by keeping adding successful cases to the case base.

- Design new RPP. When there is no reusable case in the RPP case base, which usually happens early in the system construction, a new process planning needs to be designed and then retained in the case base. As the case base gets richer, the less chance this step is needed. 


\section{Knowledge modelling for remanufacturing process planning}

Knowledge for RPP means a bunch of useful information that describes the real condition of the remanufactured cores and the corresponding sound solutions to support remanufacturing operations. The following contents detail the steps of knowledge modelling.

Although remanufacturing is an activity for EOL products, the information generated at the BOL and MOL stages, e.g. product material, geometry and specifications is crucial to the remanufacturing decision-making (Cao et al., 2009). In addition, the selling price of the new products at the BOL stage and that of the remanufactured products affect the decision-making for remanufacturability, on the other hand, the sale of remanufactured products may affect the demands for new products. The service condition at MOL will have impacts on the e.g. failure type and severity in the EOL phase, directly influence the remanufacturing processing routes and parameters. Such life cycle information of returned cores is collected for further knowledge modelling.

\subsection{Remanufacturing-relevant information extraction}

Inevitably, there will be redundant information in the collected remanufacturingrelevant information, which will interfere with the efficiency and accuracy of knowledge reuse. Thus, useful information needs to be extracted and then converted to remanufacturing-relevant knowledge to support knowledge reuse. According to their relationships with the RPP, remanufacturing-relevant information is extracted into 5 classes, including design specification, market, failure feature, process planning, and remanufacturing resource. Generally, design specification class contains basic design information of the product such as geometry, size, material and weight, product specifications; market class shows the brand, manufacturer and price of the product; failure feature class presents the failure condition of the core including failure mode, degree and location and so on; process routes and process parameters are included in the process planning class; and the remanufacturing resource class includes information such as devices and operators, supporting the execution of remanufacturing operations. Fig. 2 shows the upper relationships between the RPP and the remanufacturing-relevant information classes. It is worth noting that the design specification information, market information, and failure information are determined the input of the RPP which 
1 determines the output including the process planning and remanufacturing resource

2 information.

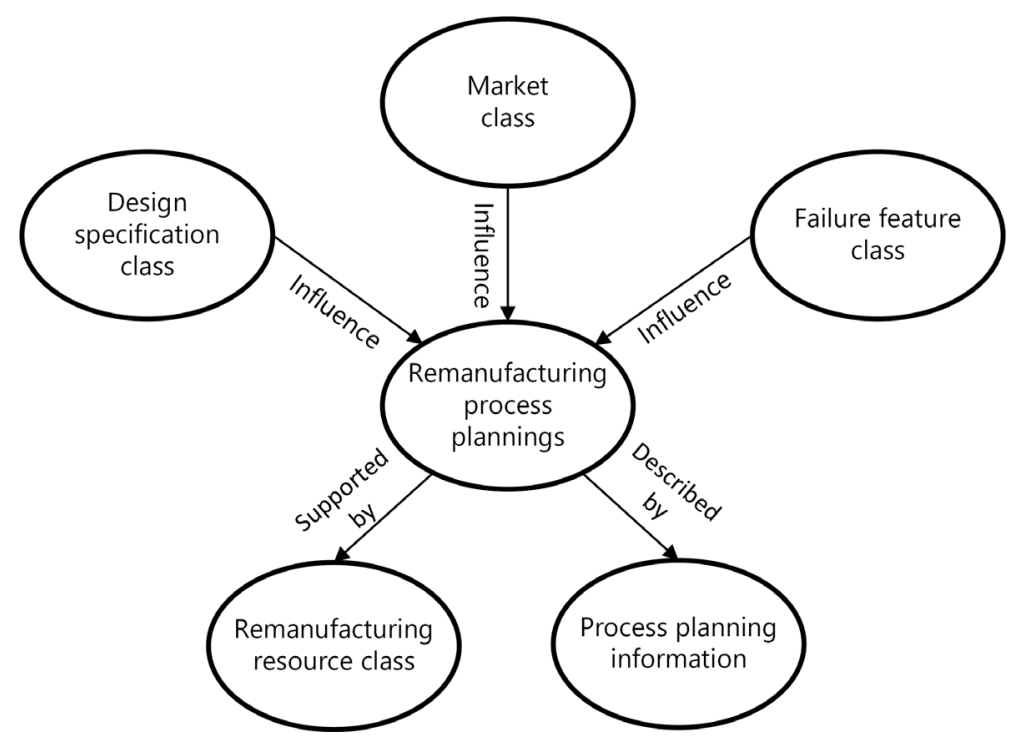

Fig. 2 Relationships between RPP and remanufacturing-relevant information classes

\subsection{Ontology based Remanufacturing knowledge construction}

On the base of previous information collection and knowledge modelling, a remanufacturing-ontology framework is proposed, which provides unified formal representations of the knowledge in the domain of remanufacturing from different sources. Fig. 3 depicts the structure of the remanufacturing-ontology. Normally, a returned core is generally made up of multiple parts which are to be disassembled during remanufacturing, but sometimes it can also be a to-be remanufactured part directly which can't be disassembled any more. In the remanufacturing-ontology framework, cores and parts are connected by a logical 'Has-part' in the ontology. Each disassembled part, such as a crankshaft, has five information classes as mentioned before. Their relationships with RPP have already been presented in Fig. 2. These information classes are composed of several attributes through a logical 'Has-a', for instance, failure feature class is composed of failure mode, failure degree, and failure location, etc. Table 1 lists the 15 attributes in RPP using crankshaft as an example including geometry, size, material, weight, journal diameters, surface roughness, surface hardness, parallelism, precision, brand, manufacturer, price, failure mode, failure degree, and failure location. These attributes are known as influence factors which are identified by skilled experts according to their importance to remanufacturing decision. In addition, the influence domain and the relevance of influence factors to 
1 remanufacturing are also given in Table 1.

2 The process planning class is composed of attributes including process routes,

3 parameters, time, cost, etc. The process route of the process planning class is an ordered

4 step of remanufacturing processes, and the corresponding process parameters in each

5 step are recorded in the process parameters attribute. The process time and cost are

6 important information that can help operators make or adjust remanufacturing strategies

7 and production scheduling in addition to decision making whether or not an EOL

8 product should be remanufactured. Similarly, remanufacturing resource class is

9 essential to the implementation of the RPP by providing necessary remanufacturing

10 resources such as device, operator, plant, and forklift.

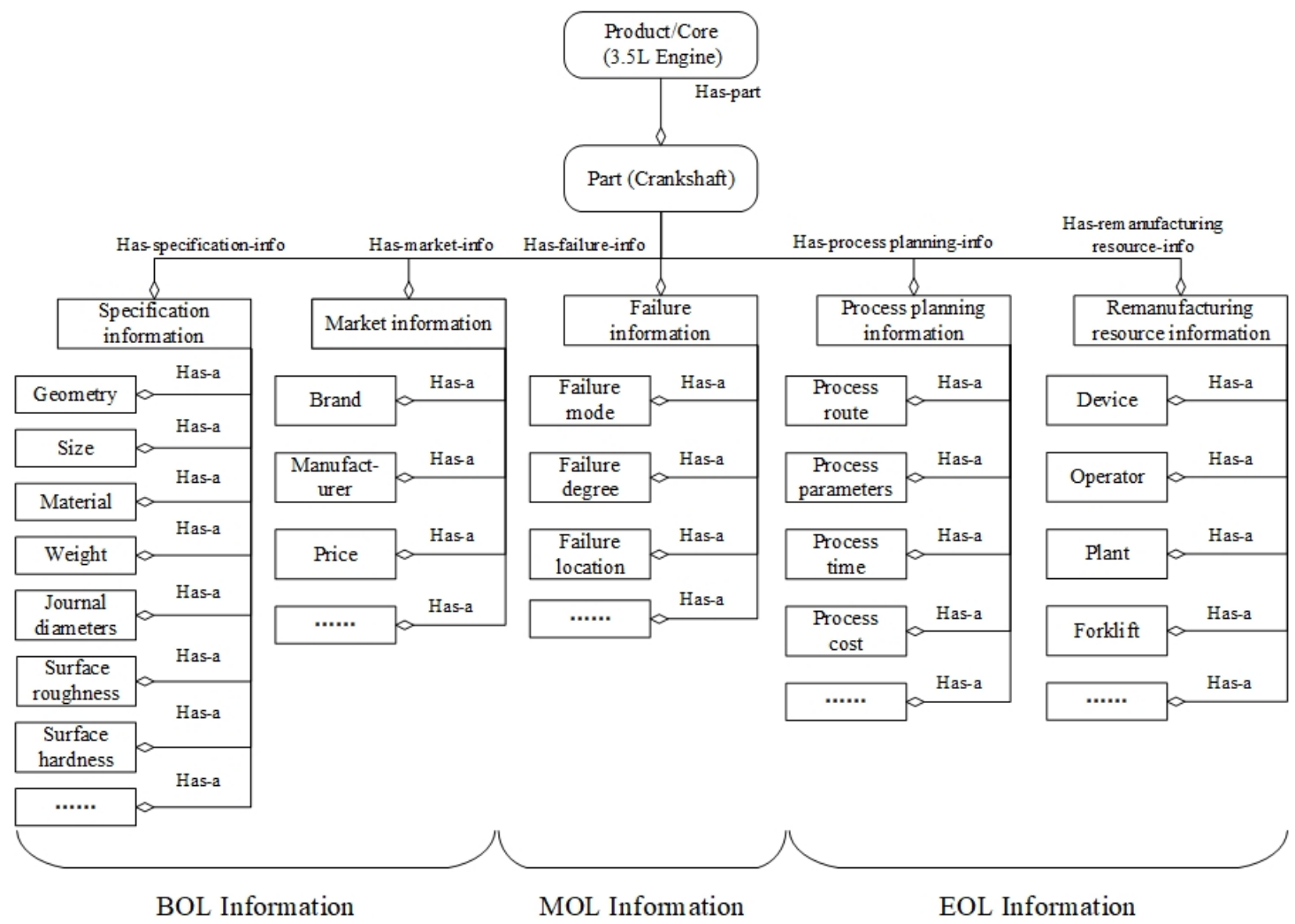

12 Fig. 3 Classes in the remanufacturing-ontology (crankshaft is an example)

13 Table 1: Influence factors related to crankshaft remanufacturing process planning.

\begin{tabular}{llllll}
\hline No. & Influence factor & Type & Label & Influence domain & Relevance to remanufacturing \\
\hline 1 & Geometry & S & S1 & Selection of process & $\begin{array}{l}\text { Milling for prismatic part and turning for } \\
\text { cylinder part for restoration of defects }\end{array}$ \\
2 & Size & S & S2 & Selection of machine & $\begin{array}{l}\text { Need to be within the threshold of machines } \\
\text { at the remanufacturing factory }\end{array}$ \\
3 & Material & S & S3 & Process parameters & $\begin{array}{l}\text { Remanufacturing processes } \\
\text { Need to be within the threshold of machines } \\
4\end{array}$ \\
Weight & S & S4 & Selection of machine & \begin{tabular}{l} 
Ne the remanufacturing factory \\
\hline
\end{tabular}
\end{tabular}




\begin{tabular}{|c|c|c|c|c|c|}
\hline 5 & Journal diameters & $\mathrm{S}$ & S5 & Process parameters & $\begin{array}{l}\text { Need to be within the threshold of machines } \\
\text { at the remanufacturing factory }\end{array}$ \\
\hline 6 & Surface roughness & $\mathrm{S}$ & S6 & Process parameters & Remanufacturing processes \\
\hline 7 & Surface hardness & $\mathrm{S}$ & S7 & Surface treatment & $\begin{array}{l}\text { Remanufacturing process route, e.g. } \\
\text { Coating or not }\end{array}$ \\
\hline 8 & Parallelism & $\mathrm{S}$ & $\mathrm{S} 8$ & Process route & $\begin{array}{l}\text { Remanufacturing process route, e.g. } \\
\text { Straightening or not }\end{array}$ \\
\hline 9 & Precision & $\mathrm{S}$ & S9 & Process parameters & $\begin{array}{l}\text { process parameters, e.g. cutting speed, } \\
\text { federate etc. }\end{array}$ \\
\hline 10 & Brand & M & M1 & Performance requirements & $\begin{array}{l}\text { Quality inspection for remanufactured } \\
\text { products }\end{array}$ \\
\hline 11 & Manufacturer & M & M2 & Performance requirements & $\begin{array}{l}\text { Quality inspection for remanufactured } \\
\text { products }\end{array}$ \\
\hline 12 & Price & M & M3 & Remanufacturability & $\begin{array}{l}\text { Different reuse strategy adapted based on } \\
\text { the price of new/remanufactured products }\end{array}$ \\
\hline 13 & Failure mode & $\mathrm{F}$ & F1 & Process route & $\begin{array}{l}\text { Process route and efficiency dependent on } \\
\text { failure modes, e.g. wear and crack }\end{array}$ \\
\hline 14 & Failure degree & $\mathrm{F}$ & $\mathrm{F} 2$ & Process route & $\begin{array}{l}\text { Process route and efficiency dependent on } \\
\text { failure degree, e.g. slight, medium, high, } \\
\text { and severe }\end{array}$ \\
\hline 15 & Failure location & $\mathrm{F}$ & F3 & Process route & $\begin{array}{l}\text { failure location will affect tool accessibility } \\
\text { for the restoration of defects }\end{array}$ \\
\hline
\end{tabular}

1 S: Design specification class

2 M: Market class

3 F: Failure feature class

$4 \quad 4.3$ Generation of the remanufacturing case semantic model

5 According to the remanufacturing-ontology, remanufacturing cases can be 6 constructed as a semantic model to make it easier to be processed. 


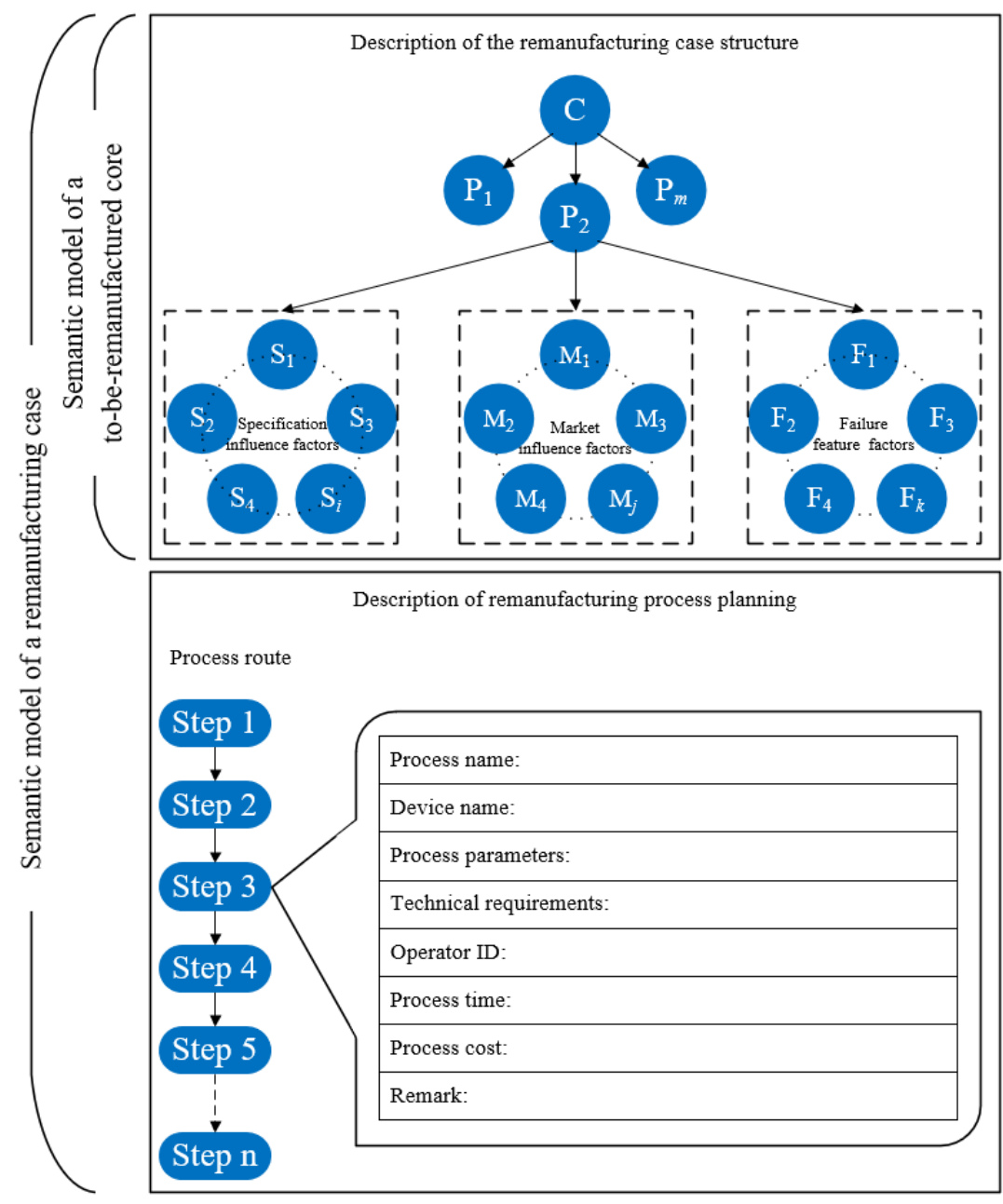

Fig. 4 Semantic model of a remanufacturing case

Fig. 4 depicts the semantic model for remanufacturing cases, which is composed of two parts: (1) the semantic description of the remanufacturing case structure; (2) the similar previous successful solution for the to-be remanufactured case (which includes process route, parameters, devices, operators, etc.).

For the semantic modeling in Fig. 4, a to-be-remanufactured core ( $\mathrm{C}$ in Fig 4) is usually disassembled to several parts (P in Fig 4) each of which can be subjected to several remanufacturing processes such as cleaning, machining, welding, coating, and reassembly depending on the condition of individual parts. A case structure contains three types of influence factors of the target to-be remanufactured part as shown in the model in Fig 4. As explained earlier, a case of the to-be remanufactured part is described by influence factors including design specifications ( $\mathrm{S}$ type), market influence (M type), and failure feature (F type) as shown in Table 1.

For the description of remanufacturing process planning in Fig. 4, a new RPP to the to-be remanufactured part is developed. As shown in Fig. 4, a process route 
1 composed of a series of process steps is employed to restore the to-be remanufactured

2 parts, and each step in the process route also has several attributes to perform the

3 specific repair actions including process name, device name, process parameters,

4 technical requirements, operator ID, process time, process cost, remark, etc.

\section{5. Algorithm to calculate local and global similarity for case retrieval}

After the remanufacturing case base has been constructed, the previous decision

7 for remanufacturing RPP is recorded and stored. The CBR method needs to be 8 implemented to retrieve and reuse the stored remanufacturing knowledge. In a CBR 9 system, the existing RPP is retrieved via the calculation of the similarity calculation.

10 The similarity can be divided into local and global similarity. The local similarity is 11 between two attributes and the global similarity is between two cases. Fig. 5 shows the 12 method for calculating local and global similarity between new case $\mathrm{X}$ and existing case $13 \mathrm{Y}$, where $i$ means the sequence number of influencing factors and $n$ represents the total 14 number of the influence factors. Once the influence factors $(\mathrm{C} 1, \mathrm{C} 2, \mathrm{C} 3 \ldots . . \mathrm{Cn})$ of the 15 to-be remanufactured case are identified in the semantic model, local similarities of 16 these attributes can be calculated by the nearest-neighbor matching method. Through 17 Analytic Hierarchy Process (AHP) method, each factor is given a weight according to 18 its importance to the case, and then the global similarity between the new case $\mathrm{X}$ and 19 case $\mathrm{Y}$ in the knowledge base can be computed considering all the local similarities and 20 their respective weights. In AHP, a pairwise comparison matrix is created based on 21 decision-maker inputs, which gives comparative importance between two influence 22 factors. Experts need to make a judgment of the factor $\mathrm{A}$ relative to $\mathrm{B}$, the more 23 important of the factor, the higher the score. The score is usually set to 1-9. If the 24 importance of A relative to $\mathrm{B}$ is set to 5, the importance of $\mathrm{B}$ relative $\mathrm{A}$ will be the 25 reciprocal of this number, $1 / 5$. The geometric mean can be calculated and normalized 26 to obtain the relative weight associated with each influence factor. The detail process 27 of the AHP method can refer to Ding et al., (2018) and Zhou et al. (2014). 


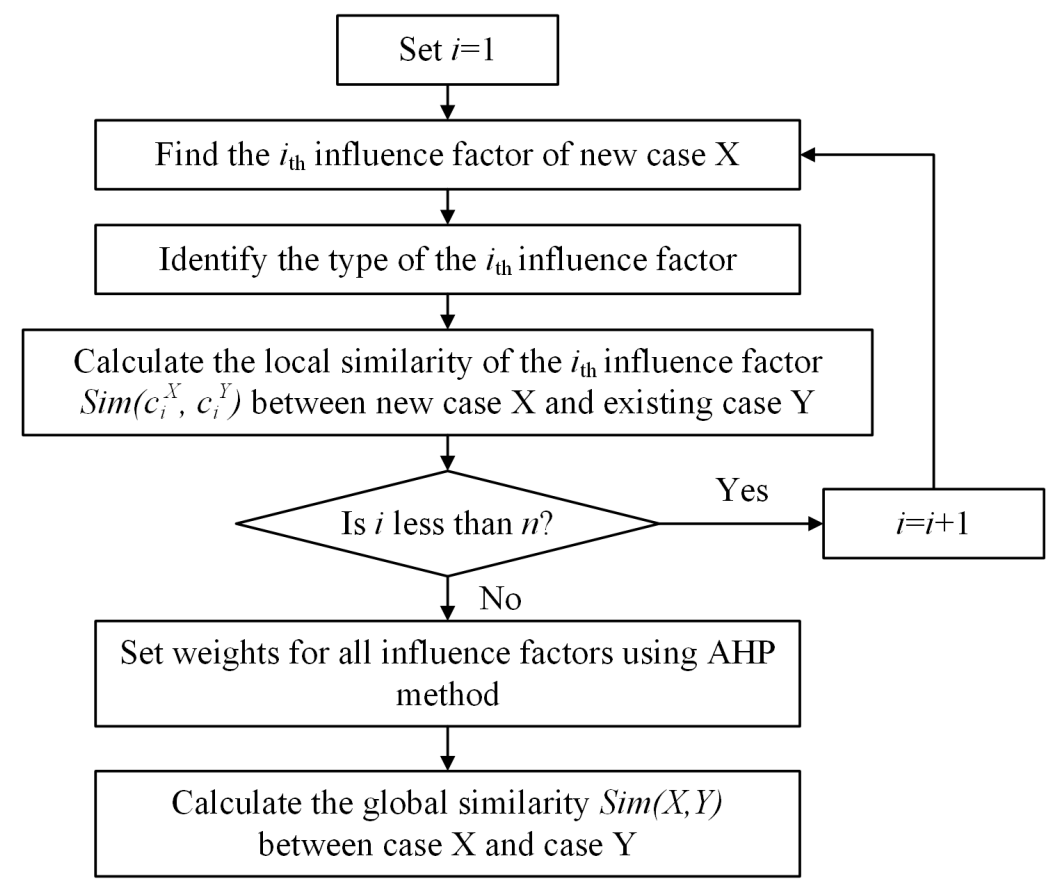

Fig. 5 Method for calculating local and global similarity to retrieve similar cases

With the local similarities calculated, the global similarity between the new case X and an existing case $\mathrm{Y}$ in the knowledge base can be calculated by (1)

.

$$
\operatorname{Sim}(X, Y)=\sum_{i=1}^{n} w\left(c_{i}\right) \operatorname{Sim}\left(c_{i}^{X}, c_{i}^{Y}\right)
$$

Where $X$ and $Y$ are the new case and existing case, respectively; $n$ is the number of influence factors. $c_{i}^{X}$ and $c_{i}^{Y}$ represent the $i_{t h}$ factors of $X$ and $Y$, and $w\left(c_{i}\right)$ is the associated important weight of this factor $c_{i} . \operatorname{Sim}(X, Y)$ is the global similarity between the new case $X$ and the existing case $Y, \operatorname{Sim}\left(c_{i}^{X}, c_{i}^{Y}\right)$ is the local similarity between $c_{i}^{X}$ and $c_{i}^{Y}$.

1 As for local similarity calculation, there are three methods for three different types of influence factors: (1) numerical factors, (2) degree factors, and (3) linguistic factors, 3 which are presented in Fig. 6.

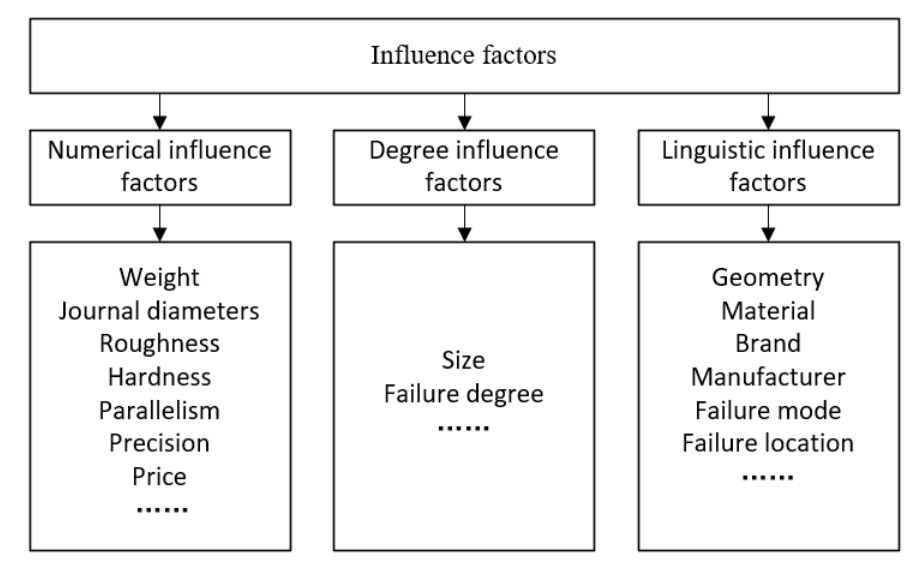

Fig. 6 Influence factors classification for local similarity calculation 
For numerical influence factors, which can be identified with the exact number, the

2 similarity can be calculated using equ. (2) where $\max \left(c_{i}\right)$ and $\min \left(c_{i}\right)$ are the maximum

3 value and the minimum value of the factor $c_{i}$ among all the cases.

$$
\operatorname{Sim}\left(c_{i}^{X}, c_{i}^{Y}\right)=1-\frac{\left|c_{i}^{X}-c_{i}^{Y}\right|}{\max \left(c_{i}\right)-\min \left(c_{i}\right)}
$$

For degree influence factors, which can be divided into different degrees after normalization, the local similarity calculation is expressed as equ. (3), where $M$ is the maximum assignment value of the factor $c_{i}$. For example, the part size is described by the ambiguous words as \{small, medium, large, huge $\}$ with the set $\{0.25,0.5,0.75,1\}$, as shown in Table 2. Similarly, the failure degree can be described by the ambiguous words as $\{$ slight, medium, high, severe $\}$ with the set $\{0.25,0.5,0.75,1\}$.

$$
\operatorname{Sim}\left(c_{i}^{X}, c_{i}^{Y}\right)=1-\frac{\left|c_{i}^{X}-c_{i}^{Y}\right|}{M}
$$

Table 2. The assignment values of different part size.

\begin{tabular}{ccccc}
\hline Degree & Small & Medium & Large & Huge \\
\hline$c i$ & 0.25 & 0.5 & 0.75 & 1 \\
\hline
\end{tabular}

For linguistic influence factors, which can't be quantified, the local similarities can be calculated using equ. (4). where local similarity is 1 if two factors are identical; otherwise the local similarity is 0 .

$$
\operatorname{Sim}\left(c_{i}^{X}, c_{i}^{Y}\right)= \begin{cases}1, & c_{i}^{X}=c_{i}^{Y} \\ 0, & c_{i}^{X} \neq c_{i}^{Y}\end{cases}
$$

\section{Case study}

Crankshaft, as a core part of the engine, is of great potential for remanufacturing (Smith and Keoleian 2004). In this section, a to-be remanufactured crankshaft of a 3.5L engine in a remanufacturing factory is taken as a case study to validate the effectiveness and feasibility of the method proposed above.

In this case, the EOL crankshaft is mainly composed of main journal, rod journal, crank arm, oil hole, the front end, and the rear end. Wear, corrosion, bending-torsion deformation and crack are the common failure modes of crankshaft. Comprehensive remanufacturing-relevant information is collected and then converted to remanufacturing-relevant knowledge. Regarding the remanufacturing ontology, nine specification influence factors, three market influence factors, and three failure feature factors are identified to describe the crankshaft remanufacturing case, specific influence 
1 factors and corresponding attributes are listed in Table 1. Meanwhile, the relative

2 importance weights of these influence factors are determined to support subsequent

3 case retrieval employing the AHP method, the results of relative importance and

4 weights are listed as shown in table 3, the labels of the influence factors have been

5 explained previously in Table 1.

6 Table 3: Importance weights of the influence factors.

\begin{tabular}{|c|c|c|c|c|c|c|c|c|c|c|c|c|c|c|c|c|}
\hline \multirow{2}{*}{$\begin{array}{l}\text { Influence } \\
\text { factors }\end{array}$} & \multicolumn{9}{|c|}{ Design specification influence factors (S) } & \multicolumn{3}{|c|}{$\begin{array}{c}\text { Market influence } \\
\text { factors (M) }\end{array}$} & \multicolumn{4}{|c|}{ Failure feature factors (F) } \\
\hline & S1 & S2 & S3 & S4 & S5 & S6 & S7 & S8 & S9 & M1 & M2 & M3 & F1 & F2 & $\mathbf{F 3}$ & Weight \\
\hline S1 & 1 & 2 & $1 / 6$ & 3 & 1 & $1 / 5$ & $1 / 5$ & $1 / 4$ & $1 / 4$ & 4 & 5 & 1 & $1 / 8$ & $1 / 8$ & $1 / 7$ & 0.0254 \\
\hline S2 & $1 / 2$ & 1 & $1 / 6$ & 1 & $1 / 2$ & $1 / 6$ & $1 / 5$ & $1 / 4$ & $1 / 4$ & 1 & 3 & 1 & $1 / 9$ & $1 / 9$ & $1 / 8$ & 0.0162 \\
\hline S3 & 6 & 6 & 1 & 6 & 4 & 1 & 1 & 2 & $1 / 3$ & 6 & 8 & 6 & $1 / 2$ & $1 / 2$ & 1 & 0.0919 \\
\hline S4 & $1 / 3$ & 1 & $1 / 6$ & 1 & $1 / 3$ & $1 / 5$ & $1 / 5$ & $1 / 5$ & $1 / 4$ & 1 & 2 & $1 / 2$ & $1 / 8$ & $1 / 8$ & $1 / 8$ & 0.0145 \\
\hline S5 & 1 & 2 & $1 / 4$ & 3 & 1 & $1 / 3$ & $1 / 2$ & $1 / 2$ & 1 & 4 & 5 & 2 & $1 / 6$ & $1 / 6$ & $1 / 6$ & 0.0343 \\
\hline S6 & 5 & 6 & 1 & 5 & 3 & 1 & 1 & 1 & 2 & 6 & 7 & 5 & $1 / 3$ & $1 / 2$ & $1 / 2$ & 0.0821 \\
\hline S7 & 5 & 5 & 1 & 5 & 2 & 1 & 1 & 1 & 1 & 5 & 6 & 3 & $1 / 4$ & $1 / 3$ & $1 / 3$ & 0.0664 \\
\hline S8 & 4 & 4 & $1 / 2$ & 5 & 2 & 1 & 1 & 1 & 1 & 5 & 7 & 3 & $1 / 5$ & $1 / 5$ & $1 / 4$ & 0.0589 \\
\hline S9 & 4 & 4 & 3 & 4 & 1 & $1 / 2$ & 1 & 1 & 1 & 5 & 5 & 2 & $1 / 5$ & $1 / 4$ & $1 / 4$ & 0.0655 \\
\hline M1 & $1 / 4$ & 1 & $1 / 6$ & 1 & $1 / 4$ & $1 / 6$ & $1 / 5$ & $1 / 5$ & $1 / 5$ & 1 & 1 & $1 / 2$ & $1 / 9$ & $1 / 8$ & $1 / 8$ & 0.0130 \\
\hline M2 & $1 / 5$ & $1 / 3$ & $1 / 8$ & $1 / 2$ & $1 / 5$ & $1 / 7$ & $1 / 6$ & $1 / 7$ & $1 / 5$ & 1 & 1 & $1 / 2$ & $1 / 9$ & $1 / 8$ & $1 / 7$ & 0.0111 \\
\hline M3 & 1 & 1 & $1 / 6$ & 2 & $1 / 2$ & $1 / 5$ & $1 / 3$ & $1 / 3$ & $1 / 2$ & 2 & 2 & 1 & $1 / 7$ & $1 / 7$ & $1 / 7$ & 0.0210 \\
\hline F1 & 8 & 9 & 2 & 8 & 6 & 3 & 4 & 5 & 5 & 9 & 9 & 7 & 1 & 1 & 2 & 0.1875 \\
\hline F2 & 8 & 9 & 2 & 8 & 6 & 2 & 3 & 5 & 4 & 8 & 8 & 7 & 1 & 1 & 2 & 0.1726 \\
\hline F3 & 7 & 8 & 1 & 8 & 6 & 2 & 3 & 4 & 4 & 8 & 7 & 7 & $1 / 2$ & $1 / 2$ & 1 & 0.1398 \\
\hline
\end{tabular}

7 To make a returned used crankshaft back to the like-new conditions a series of

8 remanufacturing processes need to be carried out. Take the crankshaft remanufacturing

9 case 1 in the case base as an example, detailed information of 9 processes is listed in

10 Table 4. Some of the process data is dummy due to the sensitivities.

11 Table 4: Detailed information of remanufacturing process planning in case 1.

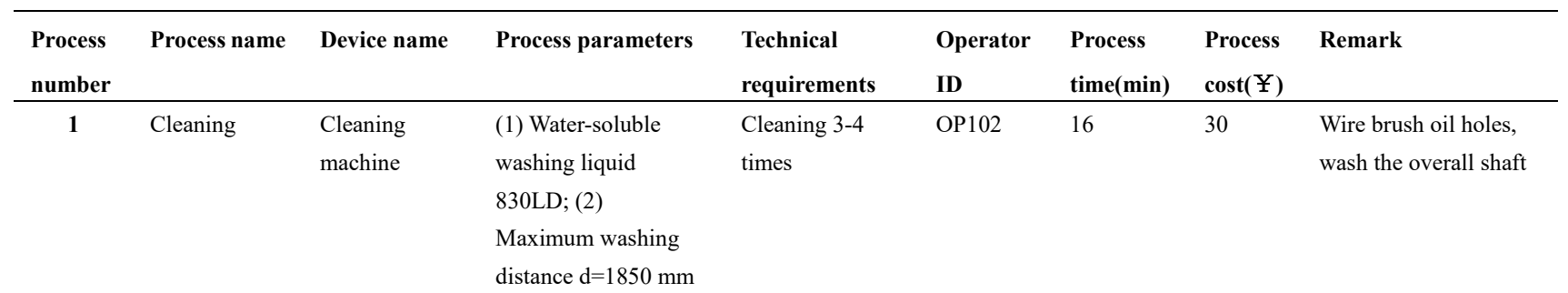




\begin{tabular}{|c|c|c|c|c|c|c|c|c|}
\hline 2 & Undercutting & $\begin{array}{l}\text { Turing } \\
\text { machine }\end{array}$ & $\begin{array}{l}\text { (1) } \mathrm{ap}=0.1 \mathrm{~mm} \\
\text { (2) } \mathrm{f}=0.03 \sim 0.07 \mathrm{~mm} / \mathrm{r} \\
\text { (3) } \mathrm{v}=15 \sim 25 \mathrm{~m} / \mathrm{min}\end{array}$ & $\begin{array}{l}\text { (1) Remove } \\
\text { excess material; } \\
\text { (2) No cracks } \\
\text { are allowed }\end{array}$ & OM201 & 20 & 40 & $\begin{array}{l}\text { Eliminate wear before } \\
\text { buildup }\end{array}$ \\
\hline 3 & Thermal spry & $\begin{array}{l}\text { Thermal spry } \\
\text { machine }\end{array}$ & $\begin{array}{l}\text { (1) Jet } \\
\text { distance }=20 \mathrm{~cm} ;(2) \\
\text { Nozzle diameter }=2 \mathrm{~cm}\end{array}$ & - & OM302 & 20 & 60 & $\begin{array}{l}\text { Important but not } \\
\text { always necessary }\end{array}$ \\
\hline 4 & Welding & $\begin{array}{l}\text { Submerged } \\
\text { arc welding } \\
\text { machine }\end{array}$ & $\begin{array}{l}\text { (1) Weld current: 900- } \\
\text { 1000 A; (2) Arc } \\
\text { voltage: 40-42 V; } \\
\text { (3) Weld speed: } 20 \\
\mathrm{~cm} / \mathrm{min}\end{array}$ & $\begin{array}{l}\text { Smooth welding } \\
\text { seam without } \\
\text { porosity }\end{array}$ & OM407 & 20 & 50 & $\begin{array}{l}\text { Dry the electrode and } \\
\text { flux before welding }\end{array}$ \\
\hline 5 & Grinding & $\begin{array}{l}\text { Grinding } \\
\text { machine }\end{array}$ & $\begin{array}{l}\text { (1) Wheel rotational } \\
\text { speed } 2800 \mathrm{r} / \mathrm{min} \text {; (2) } \\
\text { Coarse grinding } \\
\text { allowance } 0.2 \mathrm{~mm} \text {; (3) } \\
\text { Feed speed } 2 \mathrm{~mm} / \mathrm{min}\end{array}$ & $\begin{array}{l}\text { Grinding way: } \\
\text { cut mill }\end{array}$ & OM407 & 30 & 70 & $\begin{array}{l}\text { (1) Grinding wheel } \\
\text { code G80V60; (2) } \\
\text { Injection for pressure } 3 \\
\text { Mpa, fluid flow } \\
16 \mathrm{~L} / \mathrm{min}\end{array}$ \\
\hline 6 & Shot Peening & $\begin{array}{l}\text { Shot peening } \\
\text { machine }\end{array}$ & $\begin{array}{l}\text { (1) Steel shot } \\
\text { diameter: } 0.8 \mathrm{~mm} ;(2) \\
\text { Shot distance: } \\
\text { 150mm; }\end{array}$ & $\begin{array}{l}\text { The angle of } \\
\text { shot peening }> \\
40^{\circ}\end{array}$ & OM308 & 10 & 50 & $\begin{array}{l}\text { No heat treatment after } \\
\text { shot peening in general }\end{array}$ \\
\hline 7 & Polishing & $\begin{array}{l}\text { Polishing } \\
\text { machine }\end{array}$ & - & $\begin{array}{l}\text { Polished surface } \\
\text { roughness of } \\
\text { transition fillet } \\
\text { to } \mathrm{Ra} \leqslant 1.6 \text {; } \\
\text { main journal to } \\
\mathrm{Ra} \leqslant 0.4\end{array}$ & OM308 & 30 & 45 & $\begin{array}{l}\text { Polishing to technical } \\
\text { cooperation } \\
\text { requirements }\end{array}$ \\
\hline 8 & Test & $\begin{array}{l}\text { Hardness } \\
\text { testing } \\
\text { machine }\end{array}$ & - & $\begin{array}{l}\text { Standard } \\
\text { hardness } \geqslant 40\end{array}$ & OT001 & 20 & 30 & -— \\
\hline 9 & Rustproof & Spray gun & Using cosmoline & $\begin{array}{l}\text { Rustproof } \\
\text { overall } \\
\text { crankshaft }\end{array}$ & OP102 & 10 & 10 & - \\
\hline
\end{tabular}

1 After the influence factors and their relative importance weights have been

2 identified, local similarity and global similarity need to be calculated between the new

3 case $\left(\mathrm{C}_{n}\right)$ and existing cases $(\mathrm{C} 1, \mathrm{C} 2, \mathrm{C} 3, \mathrm{C} 4, \mathrm{C} 5, \mathrm{C} 6)$ in the case base to retrieve the

4 closest match from existing RPP for the new to-be remanufactured crankshaft. Detailed

5 information on these cases is shown in Table 5. Influence factors (S4, S5, S6, S7, S8,

6 S9, M3) are numerically calculated by equ. (2), (S2, F2) are degree influence factors

7 which are calculated by equ. (3), and (S1, S3, M1, M2, F1, F3) are linguistic influence

8 factors which are calculated by equ. (4) respectively. Take local similarity S4, the

9 weight of the core, between case 1 and case $n$ as an example:

$$
\operatorname{Sim}\left(c_{i}^{X}, c_{i}^{Y}\right)=1-\frac{\left|c_{i}^{X}-c_{i}^{Y}\right|}{\max \left(c_{i}\right)-\min \left(c_{i}\right)}=0.4444
$$

11 The local similarity of other factors can be obtained in the same way. Then, the 12 global similarity between case 1 and case $\mathrm{n}$ can be obtained as follows:

$$
\operatorname{Sim}(X, Y)=\sum_{i=1}^{n} w\left(c_{i}\right) \operatorname{Sim}\left(c_{i}^{X}, c_{i}^{Y}\right)=0.8196
$$


1 The results of local and global similarity are listed in Table 6. Eventually, as can be

2 seen in Table 6, $\mathrm{C} 1$ is the most similar case retrieved in the database with a similarity

3 of 0.8196 , whose RPP is most promising to be reused for the new coming arrivals. After

4 comparing case $\mathrm{C} 1$ and $\mathrm{Cn}$, the retrieved RPP is revised manually according to the real

5 situation, if necessary. After the remanufacturing processes such as cleaning,

6 undercutting, thermal spray, welding, grinding, shot peening, polishing, test, and

7 rustproof, the returned used crankshaft is restored into a new remanufactured crankshaft

8 as shown in the Fig. 7.

9 Table 5: Detailed information about existing cases and the new case.

\begin{tabular}{|c|c|c|c|c|c|c|c|c|c|c|c|c|c|c|c|}
\hline \multirow{2}{*}{$\begin{array}{l}\text { Case } \\
\text { No. }\end{array}$} & \multicolumn{9}{|c|}{$\begin{array}{c}\text { Specification influence } \\
\text { factors (S) }\end{array}$} & \multicolumn{3}{|c|}{$\begin{array}{c}\text { Market influence } \\
\text { factors (M) } \\
\end{array}$} & \multicolumn{3}{|c|}{$\begin{array}{c}\text { Failure feature } \\
\text { factors (F) }\end{array}$} \\
\hline & S1 & S2 & S3 & S4 & S5 & S6 & S7 & S8 & S9 & M1 & M2 & M3 & F1 & F2 & F3 \\
\hline $\mathrm{C} 1$ & Cylinder & Small & Cast iron & 15 & 48 & 0.8 & 45 & 0.012 & 6 & Honda & Aisin Seiki & 2000 & Wear & Slight & Main journal \\
\hline $\mathrm{C} 2$ & Cylinder & Small & Cast steel & 17 & 52 & 0.4 & 50 & 0.011 & 7 & Honda & IT Forging & 2200 & Wear & Slight & Rod journal \\
\hline $\mathrm{C} 3$ & Cylinder & Medium & Forged steel & 30 & 64 & 0.2 & 60 & 0.01 & 8 & Toyota & Aisin Seiki & 5500 & Corrosion & Medium & The front end \\
\hline $\mathrm{C} 4$ & Irregular & Medium & Cast iron & 28 & 64 & 0.8 & 45 & 0.013 & 6 & Audi & $\begin{array}{l}\text { Bharat } \\
\text { Forge }\end{array}$ & 4500 & Deformation & Slight & Crank arm \\
\hline C5 & Cylinder & Large & Cast steel & 55 & 78 & 0.8 & 50 & 0.011 & 7 & Honda & Aisin Seiki & 8200 & Wear & High & Main journal \\
\hline C6 & Cylinder & Large & Forged steel & 60 & 82 & 0.4 & 60 & 0.01 & 8 & Toyota & IT Forging & 9000 & Wear & Medium & Main journal \\
\hline Cn & Cylinder & Medium & Cast iron & 40 & 60 & 0.8 & 50 & 0.013 & 8 & Honda & Aisin Seiki & 4000 & Wear & Medium & Main journal \\
\hline
\end{tabular}

10 Table 6: Results of local similarity and global similarity.

\begin{tabular}{lccccccc}
\hline Influence factors & $\mathbf{C 1}$ & $\mathbf{C 2}$ & $\mathbf{C 3}$ & $\mathbf{C 4}$ & $\mathbf{C 5}$ & $\mathbf{C 6}$ & Weight \\
\hline Local similarity S1 & 1.0000 & 1.0000 & 1.0000 & 0.0000 & 1.0000 & 1.0000 & 0.0254 \\
Local similarity S2 & 0.7500 & 0.7500 & 1.0000 & 1.0000 & 0.7500 & 0.7500 & 0.0162 \\
Local similarity S3 & 1.0000 & 0 & 0 & 1.0000 & 0 & 0 & 0.0919 \\
Local similarity S4 & 0.4444 & 0.4889 & 0.7778 & 0.7333 & 0.6667 & 0.5556 & 0.0145 \\
Local similarity S5 & 0.6471 & 0.7647 & 0.8824 & 0.8824 & 0.4706 & 0.3529 & 0.0343 \\
Local similarity S6 & 1.0000 & 0 & 0 & 1.0000 & 1.0000 & 0.3333 & 0.0821 \\
Local similarity S7 & 0.6667 & 1.0000 & 0.3333 & 0.6667 & 1.0000 & 0.3333 & 0.0664 \\
Local similarity S8 & 0.6667 & 0.3333 & 0 & 1.0000 & 0.3333 & 0.3333 & 0.0589 \\
Local similarity S9 & 0 & 0.5000 & 1.0000 & 0 & 0.5000 & 1.0000 & 0.0655 \\
Local similarity M1 & 1.0000 & 1.0000 & 0 & 0 & 1.0000 & 0 & 0.0130 \\
Local similarity M2 & 1.0000 & 0 & 1.0000 & 0 & 0 & 0 & 0.0111 \\
Local similarity M3 & 0.7143 & 0.7429 & 0.7857 & 0.9286 & 0.4000 & 0.2857 & 0.0210 \\
Local similarity F1 & 1.0000 & 1.0000 & 0 & 0 & 1.0000 & 1.0000 & 0.1875 \\
Local similarity F2 & 0.7500 & 0.7500 & 1.0000 & 0.7500 & 0.7500 & 1.0000 & 0.1726 \\
Local similarity F3 & 1.0000 & 0 & 0 & 0 & 1.0000 & 1.0000 & 0.1398 \\
Global similarity & 0.8196 & 0.5352 & 0.3710 & 0.4832 & 0.7424 & 0.6982 & -- \\
\hline
\end{tabular}

11 

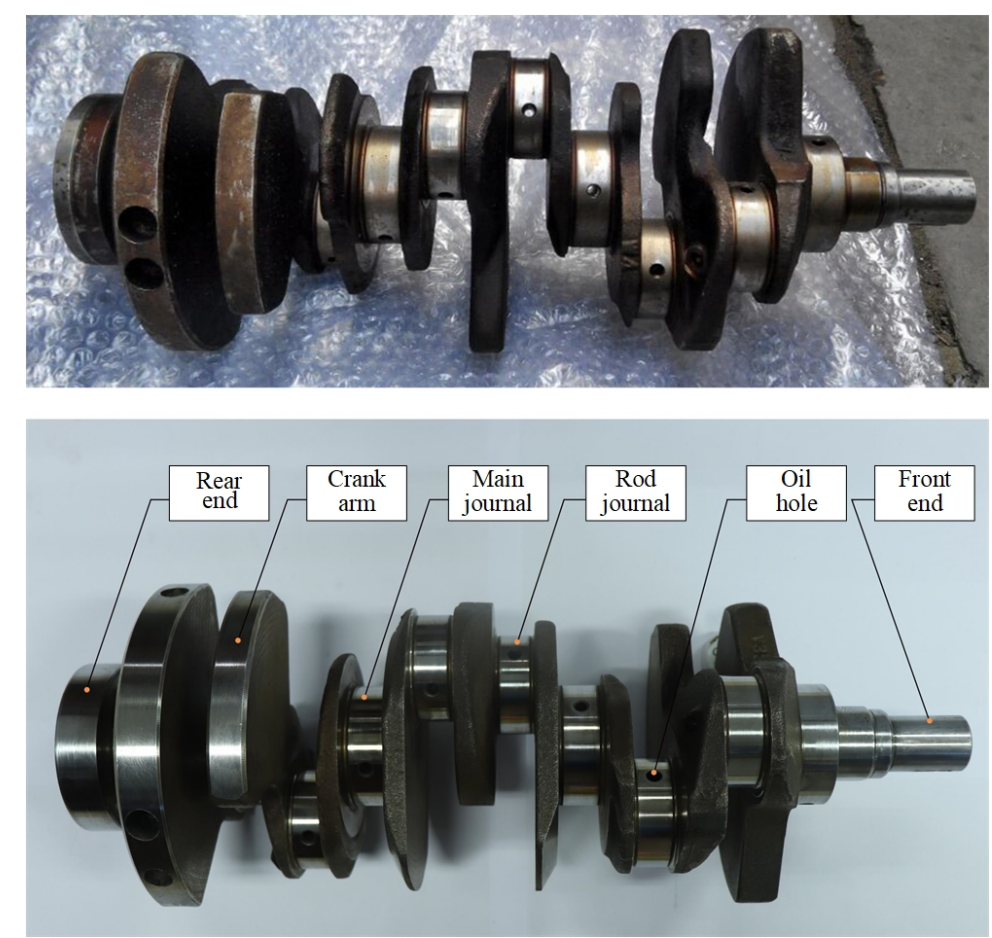

Fig. 7 The returned to-be remanufactured crankshaft and the remanufactured new crankshaft

In this case, six cases' similarities were calculated rapidly using the developed program. Significant time for case retrieval can be reduced since the developed CBR method is an intelligent and automatic process. The highest similarity retrieved is 0.8196, and when the number of cases in the case base increase, a better existing case might be retrieved. The required time and cost for remanufacturing processes are obtained in the retrieved RPP, which can facilitate the decision-making in remanufacturing practices.

Some useful managerial insights could be obtained employing the proposed method in the real-world cases. Firstly, the RPP designer can design RPP for the new incoming core by reusing the remanufacturing knowledge, improving the efficiency of remanufacturing. Besides, the remanufacturing knowledge is stored as the form of cases in this method, it is possible to effectively retrieve a very similar or even identical case when the number of cases in the case base is large enough. What's more, by introducing ontology technology into knowledge modelling, the remanufacturing relevant knowledge can be unified and shared for different domains. The knowledge accumulation and reuse will bring huge profits to the enterprise.

In this paper, to facilitate the rapid generation of RPP for new arrivals of EOL products, an interface program named 'CBR system' has been developed based on Microsoft Access 2017 to implement the proposed method. Fig. 8 shows the main form 
1 of the program; The operators need to input the necessary attributes of the case into the

2 system which will automatically calculate the local similarity and global similarity and

3 then return the most similar cases for reference. If a reusable existing remanufacturing

4 case is found, it will be used for revising if applicable for the new RPP. If there is no

5 reusable case e.g. very low similarity value, the operator needs to enter the RPP from

6 the beginning. The new generated RPP, no matter revised or redesigned, will be retained

7 into the case base to improve the problem-solving ability of the CBR system

8 continuously because the more successful remanufacturing cases are recorded, the

9 higher probability it is to retrieve reusable cases.

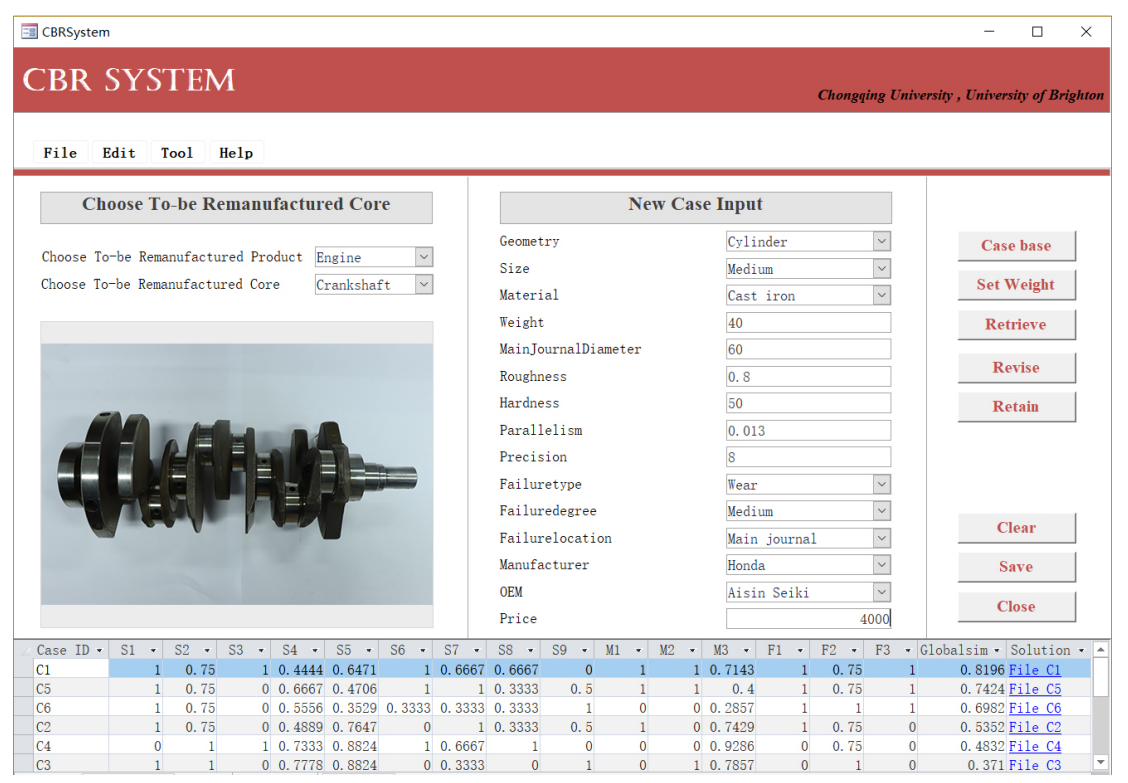

Fig. 8 The main form of application program for CBR system

\section{Conclusion and future work}

RPP is an important but complex step in the remanufacturing. Efficient RPP offers

14 great economic value and environmental benefits. The present study proposes an ontology-based method to realize the rapid generation of RPP for a to-be remanufactured core.

In this method, ontology is used to organize and formalize different kinds of remanufacturing-relevant information from different sources and life cycle stages. It

19 provides a structured way to express and manage remanufacturing knowledge in the

20 information processing system. Meanwhile, CBR is employed to retrieve and reuse the

21 existing remanufacturing knowledge to obtain candidate RPP. It is an intelligent process because it understands the remanufacturing knowledge stored in the semantic model. A 
1 case of crankshaft remanufacturing has been carried out to verify the feasibility and

2 efficiency of the proposed method. Furthermore, the method is generic and it can be

3 extended to the remanufacturing of other EOL products, e.g. electromechanical

4 products.

The developed method and application system would contribute to considerable time-saving and labor-saving through ontology-based knowledge-reusing, which in turn brings great profit. This study is based on the assumption that a returned core only has a defect, however, a returned core may have different failures at different locations in a real remanufacturing system. To enhance the flexibility and ability of the proposed method, for future work, the multi-failure mode in the to-be remanufactured core need to be further studied. In addition, remanufacturing information and knowledge is complex and difficult to be collected, uncertainty caused by the lacking of the necessary information in remanufacturing should be taken into consideration too.

\section{Acknowledgement}

This research is supported by National Natural Science Foundation of China (Grant No. 51605058, 51575072), Fundamental Research Funds for the Central Universities, China (No. 2018CDQYJX033).

\section{Reference.}

Assali, A. A., Lenne, D., \& Debray, B., 2009. Case retrieval in ontology-based CBR systems. In Annual Conference on Artificial Intelligence (pp. 564-571). Springer, Berlin, Heidelberg. https://doi.org/10.1007/978-3-642-04617-9_71

Batres, R., West, M., Leal, D., Price, D., Masaki, K., Shimada, Y., ... \& Naka, Y., 2007. An upper ontology based on ISO 15926. Computers \& Chemical Engineering, 31(5-6), 519-534. https://doi.org/10.1016/j.compchemeng.2006.07.004

British Standard Institute, BS 8887-2:2009. Design for manufacture, assembly, disassembly and end-of-life processing (MADE), Part 2: terms and definitions, 2009.

Cao, H., Folan, P., Mascolo, J., \& Browne, J., 2009. RFID in product lifecycle management: a case in the automotive industry. International Journal of Computer Integrated Manufacturing, 22(7), 616-637. https://doi.org/10.1080/09511920701522981 
Chen, S., Yi, J., Jiang, H., \& Zhu, X., 2016. Ontology and CBR based automated decision-making method for the disassembly of mechanical products. Advanced Engineering Informatics, $30(3)$, 564-584. https://doi.org/10.1016/j.aei.2016.06.005

Cooper, \& Paul. (2014). Data, information, knowledge and wisdom. Anaesthesia \& Intensive Care $\quad$ Medicine, 15(1), 44-45. https://doi.org/10.1016/j.mpaic.2016.10.006

Ding, Z., Jiang, Z., Zhang, H., Cai, W., \& Liu, Y. (2018). An integrated decision-making method for selecting machine tool guideways considering remanufacturability. International Journal of Computer Integrated Manufacturing, DOI : doi.org/10.1080/0951192X.2018.1550680

Feng, Y.T., Tian, Y.H., Zhu, Q.H., 2016. A combined input-output/decision making trial and evaluation laboratory method for evaluating effect of the remanufacturing sector development. Journal of cleaner production. 114, 103-113. https://doi.org/10.1016/j.jclepro.2014.10.048

Ghazalli, Z., \& Murata, A. (2011). Development of an AHP-CBR evaluation system for remanufacturing: end-of-life selection strategy. International Journal of Sustainable Engineering, 4(01), $2-15$. https://doi.org/10.1080/19397038.2010.528848

Goodall, P., Graham, I., Harding, J., Conway, P., Schleyer, S., \& West, A., 2015. Cost estimation for remanufacture with limited and uncertain information using casebased reasoning. Journal of Remanufacturing, 5(1), 7. https://doi.org/10.1186/s13243-015-0013-8

Guo, Y., Peng, Y., \& Hu, J., 2013. Research on high creative application of case-based reasoning system on engineering design. Computers in Industry, 64(1), 90-103. https://doi.org/10.1016/j.compind.2012.10.006

Han Wang, Zhigang Jiang, Hua Zhang, Yan Wang, Yihua Yang, Yi Li. (2019). An integrated MCDM approach considering demands-matching for reverse logistics. Journal of Cleaner Production, 208, 199-210. https://doi.org/10.1016/j.jclepro.2018.10.131

Ijomah, W. L., McMahon, C. A., Hammond, G. P., \& Newman, S. T., 2007. Development of design for remanufacturing guidelines to support sustainable manufacturing. Robotics and Computer-Integrated Manufacturing, 23(6), 712- 
719. https://doi.org/10.1016/j.rcim.2007.02.017

Ismail, H. N., Zwolinski, P., Mandil, G., \& Brissaud, D., 2017. Decision-making system for designing products and production systems for remanufacturing activities. Procedia CIRP, 61, 212-217. https://doi.org/10.1016/j.procir.2016.11.231

Jiang, Z., Ding,Z., Cai, W., \& Liu, Y., 2019. Data-drien ecological performance evaluation for remanufacturing process. Energy Conversion and Management, https://doi.org/10.1016/j.enconman.2019.111844

Jiang, Z. , Jiang, Y. , Wang, Y. , Zhang, H., Cao, H., \& Tian, G., 2019. A hybrid approach of rough set and case-based reasoning to remanufacturing process planning. $\mathrm{J}$ Intell Manuf. 30: 19 https://doi.org/10.1007/s10845-016-1231-0

Jiang, Z.., Fan, Z., Sutherland, J.W., 2014. Development of an optimal method for remanufacturing process plan selection. Int. J. Adv. Manuf. Technol. 72 (9), 15511558. https://doi.org/10.1007/s00170-014-5783-X

Kernbaum, S., Heyer, S., Chiotellis, S., \& Günther Seliger., 2009. Process planning for it-equipment remanufacturing. CIRP Journal of Manufacturing Science and Technology, 2(1), 13-20. https://doi.org/10.1016/j.cirpj.2009.07.003

Khadir, N. D. H. M. T., 2012. A case-based reasoning system based on domain ontology for fault diagnosis of steam turbines. International Journal of Hybrid Information Technology, 5(3).

Kin, S. T. M., Ong, S. K., \& Nee, A. Y. C., 2014. Remanufacturing process planning. Procedia CIRP, 15, 189-194. https://doi.org/10.1016/j.procir.2014.06.087

Kolodner, F. L., 1993. Case-based reasoning. San Francisco: Morgan Kaufmann Publisher.

Kuo, T. C., 2010. Combination of case-based reasoning and analytical hierarchy process for providing intelligent decision support for product recycling strategies. Expert Systems with Applications, 37(8), 5558-5563. https://doi.org/10.1016/j.eswa.2010.02.057

Liu, W. W., Zhang, B., Li, Y. Z., He, Y. M., \& Zhang, H. C., 2014. An environmentally friendly approach for contaminants removal using supercritical $\mathrm{CO} 2$ for remanufacturing industry. Applied Surface Science, 292, 142-148. https://doi.org/10.1016/j.apsusc.2013.11.102

Madin, J. S., Bowers, S., Schildhauer, M. P., \& Jones, M. B., 2008. Advancing ecological research with ontologies. Trends in ecology \& evolution, 23(3), 159- 
2 Parker, D., Riley, K., Robinson, S., Symington, H., Tewson, J., Jansson, K., ... \& Peck, D. (2015). Remanufacturing market study.

4 Peng, S., Li, T., Zhao, J., Guo, Y., Lv, S., Tan, G. Z., \& Zhang, H. (2019). Petri netbased scheduling strategy and energy modeling for the cylinder block remanufacturing under uncertainty. Robotics and Computer-Integrated Manufacturing, 58, 208-219. DOI: doi.org/10.1016/j.rcim.2019.03.004 cycle environmental and economic perspectives. Journal of Industrial Ecology, 8(1-2), 29. https://doi.org/10.1162/1088198041269463

11 Veerakamolmal, P., \& Gupta, S. M., 2002. A case-based reasoning approach for automating disassembly process planning. Journal of Intelligent Manufacturing,

14 Xie, X., Lin, L., \& Zhong, S. (2013). Handling missing values and unmatched features in a CBR system for hydro-generator design. Computer-Aided Design, 45(6), 963-976. https://doi.org/10.1016/j.cad.2013.02.004

17 Zhou, F., Jiang, Z.G., Zhang, H., Wang, Y., 2014. A case-based reasoning method for 18 remanufacturing process planning. Discrete Dyn. Nat. Soc. 2014 (5). http://dx.doi.org/10.1155/2014/168631 